\title{
A Look at Disk-Jet Connections in Stellar-Mass Black Holes
}

\author{
Jon M. Miller \\ The University of Michigan Department of Astronomy, 500 Church Street, Ann Arbor, MI, \\ 48109-1042,jonmm@umich.edu
}

\begin{abstract}
Connections between accretion disks and jets in accreting black holes are anticipated theoretically. In recent years, potential evidence for such connections has been emerging, most vividly in the convenient regime of stellar-mass black holes. In this contribution, various lines of evidence for disk-jet connections are briefly examined, from the standpoint of an observer focused on the role of the disk. While many lines of investigation may be promising in the future, obtaining multi-wavelength lightcurves and correlating jet flux in the radio band with physical parameters and phenomena tied to the accretion disk in X-rays may be the most direct.
\end{abstract}

\section{INTRODUCTION}

The aim of this contribution is to take a careful look at efforts to establish connections between accretion disks and jets in accreting black hole systems. Research in this area has accelerated in recent years. With a host of X-ray and radio observatories now able to bear on this problem, and as the highly flexible and unique RXTE mission nears its end, it is prudent to take stock of these advances, to ask which results are very robust and which need stronger support, and to try to suggest a course for the near future.

The aims of exploring possible disk-jet connections are many, including: understanding how and why jets are launched in accreting black hole systems, understanding how and why jets are quenched in some circumstances, learning the parameters which govern jet velocity and power, and understanding the contribution of jets to the radiative output of accreting black hole systems.

The central observational difficulty is that these issues must be addressed indirectly in actively accreting systems, since the innermost accretion flow cannot be imaged in those cases. Size scales corresponding to hundreds of gravitational radii (and below) can now be probed in Sgr A* (e.g., Shen et al. 2005) and M87 (Junor, Biretta, \& Livio 1999), but these black holes appear to accrete at very low rates, and for the purpose of understanding black hole growth phases in the early universe, understanding more active accretion phases is of greater interest. In this effort, stellar-mass black holes in the Milky Way represent a convenient regime in which to explore the processes that may be at work near to supermassive black holes. Flux-flux correlations, multi-wavelength lightcurves, spectra, and variability all have a role to play, but at times they can provide inconsistent or ambiguous clues. In the sections that follow, we discuss the extent to which observational evidence has addressed the interesting questions listed above. The sources at the center of this brief examination are the Galactic black holes GRS 1915+105, XTE J1118+480, 
Cygnus X-1, and GX 339-4; the neutron star systems Sco X-1 and Circinus X-1 are also important.

\section{LAUNCHING AND POWERING JETS}

Perhaps the easiest (and maybe the most interesting) question which observations can address is this: Is black hole spin important in powering jets? The potential around a spinning black hole is deeper than around a non-spinning black hole, and the linear velocity of the innermost stable circular orbit around a maximally-spinning black hole approaches $c$, whereas orbits around non-spinning black holes and neutron stars only approach 0.3-0.4c. If black hole spin is central to driving jets (for instance, though the Blandford-Znajek mechanism; Blandford \& Znajek 1977), jet velocities approaching $0.9 c$ should not be seen in accreting neutron star systems.

For a number of years, this separation appeared to hold. However, recent observations of the well-known neutron star binary Sco X-1 revealed jets with with a velocity of $v / c \simeq 0.6$ (Fomalont, Geldzahler, \& Bradshaw 2001). Even more recent observations of the neutron star binary Circinus X-1 may have revealed a jet with $v / c \geq 0.9$ (Fender et al. 2004). This finding may serve to indicate that black hole spin is likely not the most important ingredient in driving large-scale radio jets.

Two aspects of the Circinus X-1 result are worth examining: how certain is the nature of the compact object, and how certain is the jet velocity implied by observations? TypeI X-ray bursts were detected from the direction of Circinus X-1 long ago, which would certify Circinus X-1 as a neutron star system (Tennant, Fabian, \& Shafer 1986)). Some doubts remained, however, regarding whether or not the bursts were from a background source. The very recent detection of two "kilohertz" quasi-periodic oscillations (QPOs) in Circinus X-1 demands that the X-ray source is indeed a neutron star (Boutloukos, Wijnands, \& van der Klis 2006). Thus, all reasonable doubts about the nature of the central source are resolved. The velocity constraints obtained from Circinus X-1 differ from other sources, in that the velocity is taken from watching standing knots brighten in sequence (Circinus X-1 has a 16.6-day flux cycle), rather than tracing a single blob ejected from the central source (as per "microquasar" systems, and blobs observed in the neutron star binary Sco X-1). Only two cycles were followed, and it is possible that the brightening sequence was not phased correctly, or that fluctuations downstream do not sequence with the fluctuations of the central source.

It has been noted that the black hole binaries which have produced the most extremely relativistic radio jets have the strongest evidence for spin in X-rays (Fabian \& Miller 2002). It has also been noted that the most relativistic jets and ejection events have been seen in long-period black hole binaries (Garcia et al. 2003), which may already hint that if black hole spin is involved in powering jets, it may not be the only important parameter. A careful, high-cadence monitoring campaign on Circinus X-1 will be central to making a rigorous statement about the role of black hole spin in powering jets. 


\section{JET QUENCHING MECHANISMS}

It has been shown that in both stellar-mass and supermassive black holes, radio and Xray fluxes are correlated over many orders of magnitude at low fractions of the Eddington mass accretion rate (below 0.01-0.1 $L_{E d d}$; see, e.g., Gallo, Fender, \& Pooley 2003; Merloni, Heinz, \& Di Matteo 2003; Falcke, Kording, \& Markoff 2004; and Merloni et al. 2006). At the higher mass accretion rates observed in Seyfert AGN, radio flux appears to be greatly diminished (though weak radio activity and even resolved compact jets are seen in some cases). At high fractions of the Eddington limit and in soft, diskdominated phases, radio emission in stellar-mass black hole systems and X-ray binaries appears to be quenched (Gallo, Fender, \& Pooley 2003; Maccarone, Gallo, \& Fender 2003).

At present, the nature of this quenching is not well understood. A simple expectation, based partly on the predictions of advection dominated accretion flow models (ADAFs) might be that a filled inner disk in high luminosity states may be involved in quenching jets (Fender, Belloni, \& Gallo 2004). New work appears to rule-out this possibility: accretion disks appear to remain close to the black hole both in high luminosity and low luminosity phases alike (for $L_{X} / L_{E d d}=0.001$, at least; Miller et al. 2006; Miller, Homan, \& Miniutti 2006). It is possible that the mass accretion rate through the disk, more than the radius of the inner disk, may act to control jet production. It is also possible that a parameter like the ratio of energy dissipated in an electron-dominated corona to energy dissipated in the disk, may act to control jet production; this parameter would be at a minimum in high luminosity, disk-dominated states.

It has previously been suggested that jet production may be tied to the formation of poloidal magnetic fields (Blandford \& Payne 1982; for a recent example, see Livio, Pringle, \& King 2003). This is a particularly interesting possibility. The absence of a poloidal field in soft, disk-dominated phases, then, may inhibit jet production. This possibility is qualitatively consistent with the observation of a magnetically-driven wind in a soft disk-dominated state of the stellar mass black hole GRO J1655-40 (Miller et al. 2006b). In that source, the magnetic wind is not likely to be magnetocentrifugal (due to poloidal fields), due to its high mass outflow rate (Proga 2003). It is more likely driven by magnetic pressure from tangled fields in the disk. It has also been noted in the stellar-mass black hole GX 339-4 and black hole candidate H 1743-322 that winds appear to be active in soft, disk-dominated states, and to be absent in hard states (Miller et al. 2004, 2004b, 2006c). Moreover, the absence of wind absorption in hard phases in H 1743-322 and GX 339-4 cannot be due only to ionization; either the geometry must change or the wind must be quenched (Miller et al. 2004b, 2006c).

Thus, observations may support a picture in which jets are launched when poloidal fields can be maintained (e.g., when the ratio of energy dissipated in hard X-rays to that dissipated in the disk is above some threshold), and quenched when poloidal fields give way to toroidal fields, energy dissipation dominated by viscosity internal to the disk, and disk winds. 


\section{BROAD-BAND SPECTRAL DISTRIBUTIONS}

One important means of understanding the role of jets in accretion-powered systems to observe and monitor the broad-band spectral energy distribution, from radio up to hard X-rays. Radio imaging clearly reveals continuous jets in some cases (e.g., Cygnus X-1; Stirling et al. 2001), and rapidly moving blobs in others (e.g., GRS 1915+105; Fender et al. 1999), so it is clear that jets produce radio synchrotron emission downstream. The harder question to answer is: How much do jets contribute to higher frequency emission closer to the compact object?

In recent years, considerable effort has been devoted to trying to model the broadband spectral energy of black holes, with and without jets, in an effort to understand their role in the overall radiative output observed from black holes. A critical test case for these efforts is the black hole transient XTE J1118+480, which yielded excellent UV data due to its high galactic latitude (and therefore its low extinction). Both jetdominated emission models (Markoff, Falcke, \& Fender 2001), and broad-band models assuming an advective inner flow (McClintock et al. 2001, Esin et al. 2001), appear to give broadly reasonable descriptions of the data. The jet-dominated emission models fit the IR data slightly better than the more traditional broad-band model with an advective inner flow. However, more such broad-band spectral energy distributions are required to better differentiate these families of models.

Another regime in which jet emission might be differentiated from more traditional models with a Comptonizing corona or inner advective flow, is in the hard X-ray band (somewhat arbitrarily, above $100 \mathrm{keV}$ ). Jet emission - if primarily due to synchrotron emission - should not turn over abruptly in the hard X-ray band. Emission from a Comptonizing corona, however, should turn over at high energy, due to a decrease in the scattering cross section at energies approaching the rest mass of the electron. Some spectra appear to show that such a turn-over exists (e.g. Zdziarski \& Gierlinski 2004), which may suggest that jet emission does not extend up to the hard X-ray band. Background emission can be very high in hard X-ray bands, however, and instruments which could obtain such spectra (e.g., OSSE aboard $C G R O$ ) typically have very large fields of view. Such results must be viewed with these cautions in mind. As the INTEGRAL mission continues, and the detector performance and backgrounds are better understood, it may be possible to obtain more robust spectra above $100 \mathrm{keV}$. The Suzaku HXD may also offer sensitive hard X-ray spectra in the near future.

In fact, the situation is even more complex than described above. It is expected that hard X-ray emission should cause a reaction in the accretion disk known as disk reflection (e.g., George \& Fabian 1991, Nayakshin \& Kallman 2001, Ross \& Fabian 2005) . The most prominent results of this process are an Fe $\mathrm{K}$ emission line, and a "reflection hump" (actually due to Compton back-scattering in the disk) which peaks at approximately $30 \mathrm{keV}$, where it appears as subtle curvature in a positive flux sense. Disk reflection makes it particularly difficult to detect curvature which might be due to thermal Comptonization in a corona apart from that due to disk reprocessing - if a break in the power-law exists near to $30 \mathrm{keV}$, it may be lost due to extra curvature in this region. New jet models place additional emphasis on synchrotron self-Comptonization, not just direct synchrotron emission, which may further complicate efforts to discern jet-based emission from standard coronal emission in accreting black holes. 


\section{MULTI-WAVELENGTH LIGHTCURVES}

Multi-wavelength lightcurves may offer a more incisive means of parsing the roles of disks, jets, and/or hard X-ray coronae in creating the broad-band spectra observed from accreting black holes. In an IR, optical, UV, and X-ray variability study of the flux observed in XTE J1118+480, for instance, Hynes et al. (2003) conclude that the dominant variability mechanism is not simple disk reprocessing, and suggest that the flux may instead have a synchrotron origin. This finding is consistent with model for jet-based emission (Markoff, Falcke, \& Fender 2001), and likely inconsistent with more standard models.

Similar findings have recently been reported based on a multi-wavelength study of GX 339-4. In spectrally hard X-ray states, the optical and IR flux are observed to trace the X-ray flux very closely, in the same manner that X-ray flux and radio jet flux are correlated, without a measurable delay (Homan et al. 2005). This suggests that a large fraction of the optical and IR flux may arise via non-thermal processes in the jet, rather than due to reprocessing of X-ray flux in the outer disk.

Thus, whereas it can be difficult to distinguish jet-based emission models from more standard models based on broad-band spectroscopy, the situation appears more clear when multi-wavelength lightcurves are examined in detail. While these findings may demonstrate that jets are very important, in another regard their prominence is a hindrance: IR, optical, and UV emission which was formerly associated with the disk, may actually be due to the jet. If it is not possible to clearly distinguish jet emission from disk emission, learning the nature of disk-jet coupling in black holes will be very difficult. In this regard, searching for correlations between jet-based radio flux and X-ray phenomena tied to the disk (thermal continuum emission, Fe emission lines, QPOs) may be the best way forward.

\section{X-RAY-RADIO CONNECTIONS IN BLACK HOLES}

As noted above, a number of recent studies have clearly demonstrated a link between hard X-ray flux and radio flux in accreting black hole systems. Indeed, flux correlations hold in both stellar-mass black holes and supermassive black holes, and extend over several orders of magnitude. The correlations hold in low flux states, which are generally dominated by hard X-ray emission.

These flux-flux correlations are the foundation of many investigations into disk-jet coupling in black holes. But there is a potential problem: if the bulk of the hard Xray emission seen in black holes arises in the jet, and is not closely tied to the disk, the flux-flux correlations seen may not be disk-jet connections, but jet-jet connections. The missing link in studies of multi-wavelength fluxes, is how aspects of the X-ray flux which may be reliably assigned to the disk (not the corona or jet) vary with optical, IR, and radio flux. This may be the only clear means of understanding how the accretion inflow and outflow are related. 


\section{A Careful Look at the Disk-Jet Connection in GRS 1915+105}

The Galactic microquasar GRS $1915+105$ is well-known to both X-ray and radio observers as a bright, variable accretor. GRS $1915+105$ may be most notable for the radio-emitting blobs it sometimes launches at high velocities $(v / c \geq 0.9)$. As the source is bright and has been active for years, it is a natural target in which to explore disk-jet connections.

Perhaps the most exciting evidence for a disk-jet connection in GRS $1915+105$, if not in accreting black holes in general, was presented by Belloni et al. (1997). In a particular observation made with $R X T E$, dramatic swings in the source intensity and hardness over $\sim 1000 \mathrm{~s}$ spans can be explained in terms of rapid changes in the inner accretion disk radius. This behavior is highly suggestive of a disk which is periodically being "emptied". Pooley \& Fender (1997) discovered a link between this apparent behavior and radio flaring, indicating that the disk might be periodically ejected in a jet.

In order to test this connection, we analyzed the same RXTE dataset considered in Belloni et al. (1997). Using simple count-rate selections, we made average spectra from the high ("filled-disk") and low ("emptied disk") phases in the 2.8-20.0 keV band. All layers of all active PCUs were combined to create the spectra. Responses were generated using the standard "pcarsp" tool. The spectra were fit jointly using XSPEC version 11. We fit the spectra with a simple model consisting of Galactic absorption, an accretion disk (using the simple "diskbb" model), and Comptonization (using the "CompTT" model). Within this model, the seed temperature in the Comptonization model was fixed to the disk temperature.

With this simple spectral model, we find that the apparent ejection episodes can be instead described in terms of a disk which remains at the innermost stable circular orbit, and a variable corona. A disk with a color temperature of $k T=1.29(1) \mathrm{keV}$ and an apparent radius of $2 r_{g}$ (where $r_{g}=G M / c^{2}$, and assuming $d=11 \mathrm{kpc}$, an inclination of 70 degrees, and a mass of 14 solar masses) with a $k T_{e}=50 \mathrm{keV}$ Comptonizing corona changing between $\tau<0.05$ in high phases and $\tau=0.29(1)$ in low phases gives an acceptable fit to the data $\left(\chi^{2} / v=104 / 86\right)$. These results suggest that the corona, not the disk, might be periodically ejected in GRS $1915+105$. A similar conclusion was reached by Vadawale et al. (2003) using a more complex spectral model.

Our spectral results from GRS $1915+105$ are not unique; a variable disk as per Belloni et al. (1997) can also describe the variability acceptably. Our results only serve to indicate that present evidence for a disk-jet connection in GRS 1915+105 is not definitive. This case may serve as an example of the various observational challenges in clearly demonstrating a disk-jet connection. RXTE has a lower energy bound of $\sim 3 \mathrm{keV}$ and low energy resolution, which can complicate studies of accretion disks. Moreover, GRS $1915+105$ is viewed through a very high Galactic column density, which further complicates studies of the disk. 


\section{JETS AND X-RAY TIMING}

$\mathrm{X}$-ray power spectral components are believed to be tied to the disk, and can therefore be an important tool for testing disk-jet connections. There are subtle complications, however. For instance, the rms power in such components is positively correlated with $\mathrm{X}$-ray hardness (e.g., Belloni et al. 2005). Thus, the energy dependence of timing components is not disk-like, but corona-like. Within the context of this article, then, where the necessity of establishing jet correlations with true disk parameters is emphasized, it is worth briefly discussing why it is believed that QPOs are disk phenomena.

It is likely that the disk supplies frequencies which may be more vividly expressed in a corona. The best evidence for this may be come from the behavior of QPOs observed in Sco X-1: the exact modulation mechanism of $\mathrm{kHz}$ QPOs in neutron stars is not known, but the frequencies of $\mathrm{kHz}$ QPOs make it very likely that these QPOs reflect the orbital frequency of the inner disk and an interaction with the stellar surface (e.g. Barret, Olive, \& Miller 2006). Yu, van der Klis, \& Jonker (2001) showed that the properties of these $\mathrm{kHz}$ QPOs vary with the phase of slower oscillations. This finding suggests that lowfrequency QPOs, while not orbital frequencies, are still disk frequencies, perhaps due to precession or a global disk oscillation.

Similarly, the frequencies of the fastest QPOs observed in stellar-mass black hole systems is very close to Keplerian orbital frequencies expected at the innermost stable circular orbit. Once again, the nature of lower frequency QPOs is less clear, but indirect evidence suggests they are also tied to the disk. For instance, Miller \& Homan (2005) showed that the properties of broad Fe lines - believed to originate in the inner disk depend on the phase of strong low-frequency QPOs in GRS 1915+105. As with the Yu result, this suggests that low-frequency QPOs may be a global oscillation or a precession frequency.

Migliari, Fender, \& van der Klis (2005) conducted the first study of X-ray timing components and radio jet flux in the black hole GX 339-4, and a number of neutron star binaries. The exciting result of this study is that radio luminosity appears to be positively correlated with one Lorentzian component and a frequency break, and anticorrelated with the rms power in that specific Lorentzian, in GX 339-4 (individually; the same holds true for the collection of less-observed neutron star sources when considered together).

This correlation is very suggestive of a link between frequencies in the accretion disk and power in the radio jet. Two caveats must be noted: First, the study correlates the properties of Lorentzian power components and frequency breaks in the X-ray flux with the radio flux; while it is likely that broad Lorentzian components are related to QPOs, they are not properly QPOs (the term "QPO" is typically reserved for Lorentzian components for which $v / F W H M \geq 2$ ). Second, the Lorentzian with properties found to correlate with radio luminosity is just one of a few Lorentzians required to fit the X-ray power spectra.

The main advantage and disadvantage of correlations between radio parameters and $\mathrm{X}$-ray timing parameters is that the timing components are phenomenological. The timing components do not suffer from the same degree of model degeneracy that complicates disk-jet connections in GRS 1915-105 using continuum spectra. However, the fact that the precise origin of power spectral components in accreting systems is still not 
understood prevents these correlations from providing clearer insights.

\section{FUTURE DIRECTIONS}

As discussed above, multi-wavelength lightcurves have clearly shown that jets may be very important in accreting black hole systems. To reveal any coupling between the accretion disk and jet, however, more detailed work is required. It is also clear that we must move away from simply exploring radio correlations with broad-band X-ray flux, or hard X-ray flux.

In the case of stellar-mass black holes, the accretion disk can be revealed in three ways: through thermal blackbody emission, through (broad) Fe emission lines, and through X-ray timing features. Of these, our brief analysis of GRS $1915+105$ suggests that thermal continuum emission may be the most ambiguous of the potential disk diagnostics. The accretion disk reflection spectrum - most vividly seen in Fe emission lines (which are relatively independent of the continuum, see Miller et al. 2006) - is a much better diagnostic. X-ray timing components may also be good disk diagnostics, particularly if new studies can employ QPOs rather than broader Lorentzian features.

For significant progress, it is likely that as many as 100 points from which radio flux, radio spectral index, Fe line properties, and X-ray timing properties can me measured simultaneously, over the course of an outburst. This is a high standard, to be sure, but to really understand disk-jet connections, we must be able to probe beyond simple mass accretion rate variations. A large number of points will provide the ability to test whether a given correlation is statistically better than broad-band flux-flux correlations (taking flux as an imperfect indicator of mass accretion rate).

These studies will require more total exposures, and more frequent monitoring of outbursts with missions like Chandra, XMM-Newton, Suzaku, and Swift, and more focused studies with RXTE in its final years of operation. While RXTE can constrain the basic parameters of Fe lines, missions with lower background and higher energy resolution are better suited. RXTE is, however, ideally suited to provide the timing measurements required.

\section{ACKNOWLEDGEMENTS}

I thank Jeroen Homan and Simone Migliari for helpful discussions. I thank Philip Hughes, Joel Bregman, and the University of Michigan for arranging an excellent conference.

\section{REFERENCES}

Barret, D., Olive, J., \& Miller, M., 2006, MNRAS, in press

Belloni, T., et al., 2005, A\&A, 440, 207

Blandford, R., \& Payne, D., 1982, MNRAS, 199, 883

Blandford, R., \& Znajek, R., 1977, MNRAS, 179, 433 
Belloni, T., Mendez, M., King, A. R., van der Klis, M., \& van Paradijs, J., 1997, ApJ, 479, L145

Boutloukos, S., Wijnands, R., \& van der Klis, M., 2006, ATEL 695

Esin, A., et al., 2001, ApJ, 555, 483

Fabian, A., \& Miller, J., 2002, Science, 297, 947

Falcke, H., Kording, E., \& Markoff, S., 2004, A\& A, 414, 895

Fender, R., et al., 1999, MNRAS, 304, 865

Fender, R., et al., 2004, Nature, 427, 222

Fender, R., Belloni, T., \& Gallo, E., 2004, MNRAS, 335, 1105

Fomalont, E., Geldzahler, B., \& Bradshaw, C., 2001, ApJ, 553, L27

Gallo, E., Fender, R., \& Pooley, G., 2003, MNRAS, 344, 60

Garcia, M., Miller, J., McClintock, J., King, A., \& Orosz, J., 2003, ApJ, 591, 388

George, I., \& Fabian, A., 1991, MNRAS, 249, 352

Homan, J., Buxton, M., Markoff, S., Bailyn, C., Nespoli, e., \& Belloni, T., 2005, ApJ, 624, 295

Hynes, R., et al., 2003, MNRAS, 345, 292

Junor, W., Biretta, J., \& Livio, M., 1999, Nature, 401, 891

Livio, M., Pringle, J., \& King, A., 2003, ApJ, 593, 184

Maccarone, T., Gallo, E., \& Fender, R., 2003, MNRAS, 345, L19

Markoff, S., Falcke, H., \& Fender, R., 2001, A\&A, 372, L25

McClintock, J., et al., 2001, ApJ, 555, 477

Migliari, S., Fender, R., \& van der Klis, M., 2005, MNRAS, 363, 112

Miller, J. M., et al., 2004, ApJ, 601, 450

Miller, J. M., et al., 2004b, ATEL 221

Miller, J., \& Homan, J., 2005, ApJ, 618, L107

Miller, J. M., et al., 2006, ApJ, subm., astro-ph/0602633

Miller, J. M., et al., 2006b, Nature, 441, 953

Miller, J. M., et al., 2006c, ApJ, in press

Miller, J. M., Homan, J., \& Miniutti, G., 2006, ApJ, subm., astro-ph/0605190

Merloni, A., Heinz, S., \& Di Matteo, T., 2003, MNRAS, 345, 1057

Merloni, A., Kording, E., Heinz, S., Markoff, S., Di Matteo, T., \& Falcke, H., 2006, NewA, 11, 567

Nayakshin, S., \& Kallman, T., 2001, ApJ, 546, 406

Pooley, G. G., \& Fender, R. P., 1997, MNRAS, 292, 925

Proga, D., 2003, ApJ, 585, 406

Ross, R., \& Fabin, A., 2005, MNRAS, 358, 211

Shen, Z., Lo, K., Liang, M., Ho, P., \& Zhao, J., 2005, Nature, 438, 62

Stirling, A., Spencer, R., de la Force, C., Garrett, M., Fender, R., \& Ogley, R., 2001, MNRAS, 327, 1273

Tennant, A., Fabian, A., \& Shafer, R., 1986, MNRAS, 221, 27

Vadawale, S. V., Rao, A. R., Naik, S., Yadav, J. S., Ishwara-Chandra, C. H., Pramesh Rao, A., \& Pooley, G. G., 2003, ApJ, 597, 1023

Yu, W., van der Klis, M., \& Jonker, P., 2001, ApJ, 559, L29

Zdziarski, A., \& Gierlinski, M., 2004, PThPS, 155, 99 